- Leistungsfähiges Breitbandnetz

- Intelligente Netze

- Digitalisierung der deutschen In-dustrie

- Wachstum durch smarte Techno-logien

- Vernetzte und transparente Ver-waltung

- Vertrauen und Sicherheit

Der Wortlaut der Erklärung mit Erläuterungen zu den Handlungs-

feldern ist auf der Website http://www.it-gipfel.de/zu finden.

\section{Smart Meter - Smarter Datenschutz in intelligenten Stromnetzen}

Anlässlich der aktuellen Diskussion über die technische Gestaltung von intelligenten Stromzählern - Smart Meter - legt der Bundesbeauftragte für den Datenschutz und die Informationsfreiheit am Tag des Sechsten Nationalen IT-Gipfels ein Positionspapier zu den Datenschutzanforderungen an Smart Meter vor.

Smart Meter und Datenschutz

Der Bundesbeauftragte für den Datenschutz und die Informationsfreiheit (BfDI) begrüßt eine nachhaltige und effiziente Energieversorgung. Jedoch darf diese nicht zum gläsernen Energieverbraucher führen. Anlässlich der aktuellen Diskussion über die technische Gestaltung von Smart Meter, abgebildet im Schutzprofil des Bundesamtes für Sicherheit in der Informationstechnik, weist der BfDI nachdrücklich auf folgende Punkte hin:

\section{Datenschutz als Erfolgskriterium für Akzeptanz}

Der Erfolg von Smart Meter ist unmittelbar abhängig von einer hohen Akzeptanz der Verbraucher. Die Gewährleistung eines angemessenen Datenschutzes stellt dabei das wesentliche Erfolgskriterium dar. Dies belegen Meldungen aus den Niederlanden und Kanada beispielhaft: Hier hatte die Nichtbeachtung von Datenschutzund Datensicherheitsaspekten in einer frühen Phase zu einer massiven Ablehnung der neuen Stromzähler bei der Bevölkerung geführt.

\section{Lokale Datenhaltung im Smart Meter}

Daten, die von Smart Metern erhoben werden, sollen nur dann den Haushalt verlassen, wenn dies erforderlich ist. Ansätze, bei denen Verbrauchsdaten hoch aufgelöst von einer zentralen Stelle gespeichert, aufbereitet und weitergeleitet werden, und bei denen erst in der zentralen Stelle geprüft wird, ob die Daten zur Verarbeitung überhaupt erforderlich sind, lehnt der BfDI ab. Derartige zentrale Datensammlungen widersprechen den Grundsätzen der Datensparsamkeit, Datenvermeidung, Erforderlichkeit und Zweckbindung.

\section{Lokale Umsetzung variabler Tarife im Smart Meter}

Zur Umsetzung variabler Tarife sollen Smart Meter wirklich intelligent sein und die notwendigen Berechnungen zur Verbrauchsermittlung in Tarifzonen selbst durchführen. Nur so kann verhindert werden, dass der Verbraucher detaillierte Verbrauchsdaten zur Nutzung neuer Tarife preisgeben muss. Davon zu unterscheiden sind die Ermittlung der Rechnungsbeträge, die Rechnungserstellung sowie der Rechnungsversand, die auch außerhalb des Smart Meter erfolgen können.
Lokale Verbrauchsvisualisierung beim Verbraucher

Mit einer lokalen Schnittstelle von Smart Meter zum Verbraucher soll sich jeder Verbraucher ein Bild über seinen Energieverbrauch machen, ohne dass sensible Verbrauchsdaten im Sekundentakt an Dritte weitergeleitet werden müssen. Nur so hat der Verbraucher die Möglichkeit, sich an den Energieeffizienzmaßnahmen zu beteiligen, ohne datenschutzrechtliche Kollateralschäden in Kauf nehmen zu müssen.

\section{Zum Hintergrund}

Der BfDI hat sich bereits frühzeitig für bereichsspezifische gesetzliche Vorgaben eingesetzt, die die Erhebung, Verarbeitung und Nutzung der durch Smart Meter erhobenen Daten regeln. Hierbei fordert er:

- Strikte Zweckbindung der anfallenden Daten

- Nutzung personenbezogener Daten nur soweit erforderlich

- Grundsatz der Datensparsamkeit

- Transparente Information über die Datenverarbeitungstatbestände

- Datenhoheit beim Verbraucher (z.B. bei Fernmessen und Fernwartung)

- Verbindliche Standards für den technischen Datenschutz sowie die IT-Sicherheit

- Wahlfreiheit für datenschutzfreundliche Lösungen (Verbrauchsvisualisierung, Umsetzung variabler Tarife, lokale Datenhaltung beim Verbraucher)

Der Detaillierungsgrad der Daten birgt datenschutzrechtliche Risiken. Die Möglichkeit von differenzierten und engmaschigen Nutzungs- und Verhaltensprofilen in den Haushalten schafft ein großes Ausforschungspotenzial. Ein gläserner Energiekunde bzw. -nutzer muss vermieden werden. Datenschutzrechtlich problematisch stellen sich auch die neuen Rollen bei Energienutzung, Lieferung und Abrechnung dar. Neue Akteure bedeuten, dass mehr personenbezogene Daten als bisher verarbeitet und genutzt werden. Damit steigt das Fehler- und Missbrauchsrisiko.

\section{IT-Gipfel 2011 - Impuls für E-Justice}

Eine anwenderfreundliche Kommunikation mit der Justiz soll sowohl per De-Mail als auch über das elektronische Gerichts- und Verwaltungspostfach (EGVP) bundeseinheitlich möglich werden. Die Bundesministerien des Innern und der Justiz verständigen sich auf eine enge Zusammenarbeit in den Bereichen E-Government und E-Justice.

Bundesjustizministerin Sabine Leutheusser-Schnarrenberger: „Justiz und Verwaltung müssen sich an die moderne Lebenswirklichkeit anpassen. Der elektronische Rechtsverkehr und eine elektronische Aktenführung machen die Kommunikation zwischen Bürgern und Behörden einfacher, effektiver und langfristig kostengünstiger. Dies ist der richtige und wichtige Schritt in die Zukunft."

In der Justiz basiert die Kommunikation zwischen Bürger, Rechtsanwälten und Gerichten bisher fast ausschließlich auf Papier. Ein Grund hierfür ist die fehlende Akzeptanz der - für den formgerechten Zugang notwendigen - qualifizierten elektronischen Signatur. Außerdem können elektronische Dokumente bei Gericht noch immer nicht flächendeckend über das EGVP eingereicht werden. Das neu eingeführte De-Mail-System kann einen zusätzlichen sicheren Kommunikationsweg für E-Justice bieten. 